\title{
UPGRADE OF THE AO PHOTOINJECTOR LASER SYSTEM FOR NML ACCELERATOR TEST FACILITY AT FERMILAB
}

\author{
J. Ruan, H. Edwards, R. P. Fliller III, J. K. Santucci, FNAL, Batavia, IL 60510, U. S. A.
}

\begin{abstract}
The current Fermilab A0 Photoinjector laser system includes a seed laser, a flashlamp pumped multipass amplifier cavity, a flashlamp pumped 2-pass amplifier system followed by an Infra-Red (IR) to Ultra-Violet (UV) conversion stage. However the current system can only deliver up to 800 pulses due to the low efficiency of $\mathrm{Nd}$ :Glass used inside multi-pass cavity. In this paper we will report the effort to develop a new multi pass cavity based on Nd:YLF crystal end-pumped by diode laser. We will also discuss the foreseen design of the laser system for the NML accelerator test facility at Fermilab.
\end{abstract}

\section{INTRODUCTION}

The laser system for the Fermilab-A0 photoinjector has been recently upgraded in order to improve the reliability of the system and to reduce amplitude fluctuations. [1] The stability of the current laser system is greatly improved compared to the previous version. However our study shows that flashed lamp pumped Nd:Glass Multipass (MP) cavity is still the dominant noise source for the entire laser system. In particular the instability of the pumping light, i.e. the flash lamp pump scheme, is the bottle neck to improve the laser system further. Another disadvantage of the current MP cavity design is the low efficiency of the gain medium, Nd:Glass, used inside the MP structure. This prevents us realizing a long pulse train operation (up to 1000 individual pulses with $1 \mu$ s apart or 3000 pulses with 330 ns apart) which is essential for the new accelerator test facility at Fermi lab.

Neodymium-doped yttrium-lithium fluoride (Nd:YLF) is a good candidate to address this issue. It is a very efficient material that can be pumped by either flash lamp [2] or diode laser. [3] In addition the induced emission cross section is large enough to produce a single pass amplification up to 10. [2] At the same time we will also upgrade our flash lamp pump to fiber coupled laser diode pump to get better stability and higher reliability. Using optical fiber to deliver the pump to the end-pumped active medium has several practical advantages:

- The pump beam at the end of the fiber has a high quality, central symmetrical profile

- The radial size of the beam can be easily scaled up or scaled down with high quality optics

- A fiber connection provides a simple and virtually lossless interface between the pump source and the active medium
- Both the pump source and active medium can be changed simply by reconnecting the fiber between them.

Therefore it seems very attractive to use the fiber coupled diode laser to end pump the Nd:YLF crystal for our new MP cavity.

In this article we report on the progress towards our upgrade plan, i.e. the effort to build an efficient Nd:YLF Laser operated at $1053 \mathrm{~nm}$ that was end pumped by fiber coupled, high power laser diode.

\section{EXPERIMENTS}

Two laser diodes from Dilas Inc. are used in our experiment. Each of them can output up to $32 \mathrm{~W}$ at 805 $\mathrm{nm}$ using a $400 \mu \mathrm{m}$ fiber. In our applications a pair of laser control modules is used to operate the laser diode in pulsed mode. At the transport end of the fiber the laser diode radiation is imaged with $2.5 \mathrm{x}$ amplification to the end of the Nd:YLF rod. A pair of high reflective mirror at $805 \mathrm{~nm}(>99.5 \%)$ is used to reflect the pump beam into the rod. Because this mirror will be inside our cavity structure another anti-reflection (AR) coating is applied to realize high transmission (> 95\%) at $1053 \mathrm{~nm}$. During the course of our experiment we investigate the a Nd:YLF
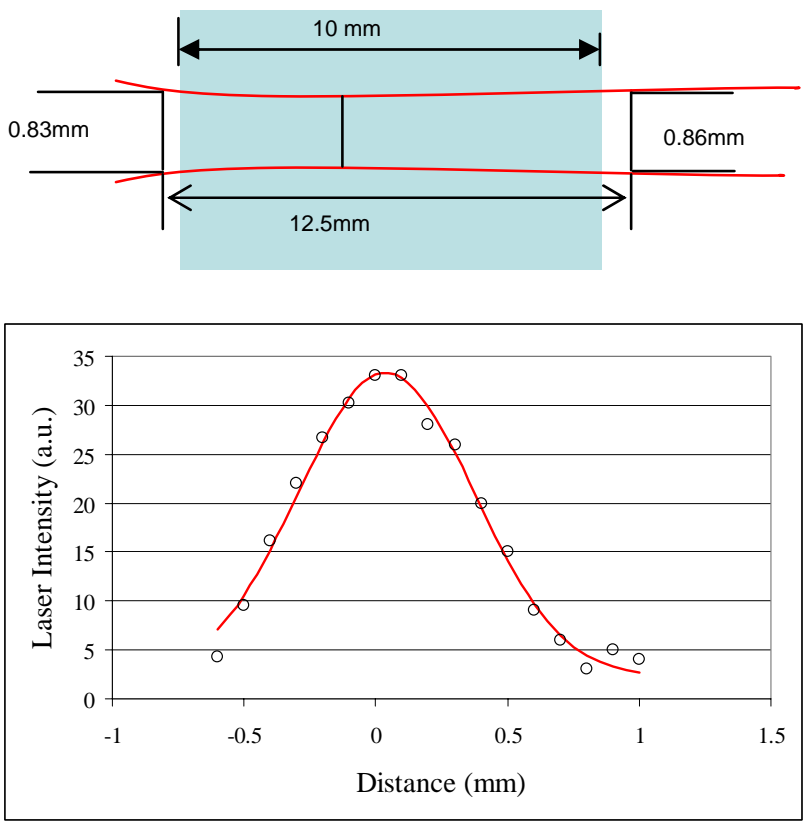

Figure 1: Pump beam size are shown throughout the YLF crystal (blue region) on the top of the figure. A typical cross section data (dots) is taken at the center of YLF crystal and fitted using gaussian profile with a FWHM of $0.8 \mathrm{~mm}$. (red solid line) 

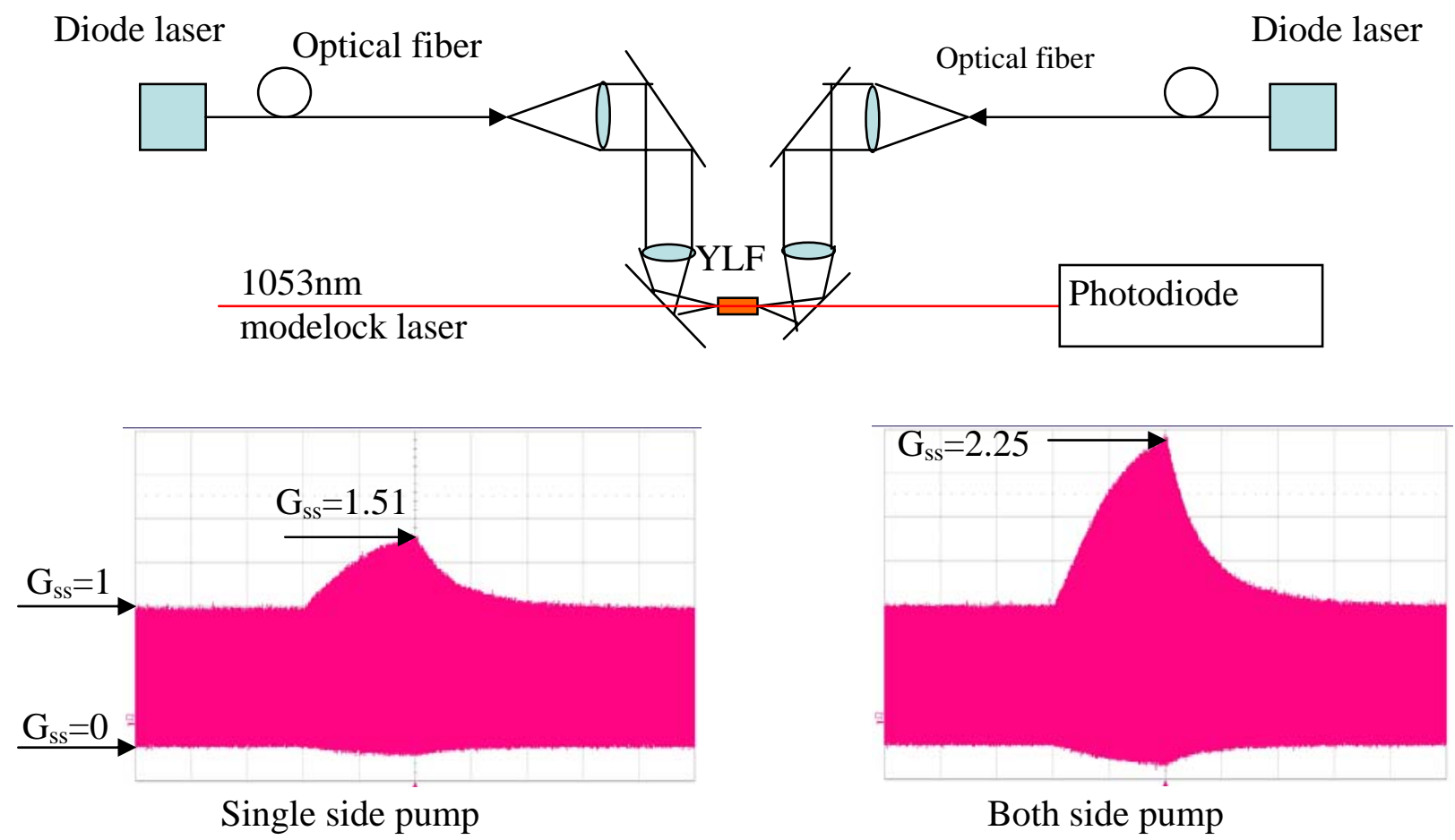

Figure 2.Single pass small gain measurement: Setup and experiment results

rod pumped from one side or both side. The active laser medium is a $4-\mathrm{mm}$ diameter and $10 \mathrm{~mm}$ long Nd:YLF rod. Both ends are AR coated for both $805 \mathrm{~nm}$ and $1053 \mathrm{~nm}$. The focused pump beam formed a circular symmetric spot at the input surface of Nd:YLF rod with a pump beam cross section of $<1 \mathrm{~mm}$ full width half maximum (FWHM) over the entire crystal length as shown at the top of figure 1. A typical horizontal profile is shown at the bottom of figure 1 . The vertical profile is almost identical to the horizontal profile as expected. More than $80 \%$ of the pump energy was absorbed in the Nd:YLF rods.

\section{Single pass small gain measurement}

In order to determine the gain of end pumped $\mathrm{Nd}: Y L F$ crystal, a cw mode-locked laser operated at $1053 \mathrm{~nm}$ is used. The laser is collimated and apertured to get the probe beam with the size of $1 \mathrm{~mm}$. The top of the figure 2 is the sketch for the experiment setup. A digital oscilloscope is used to record the waveform. The results from single side pump and both side pump are shown at the bottom of the figure 2 . The measured small signal gain is 1.51 from single side pump and 2.25 from both side pump. The pump pulse is $1.1 \mathrm{~ms}$ long and the buildup time is about $1 \mathrm{~ms}$ for our experiment.

\section{Free running end-pumped Nd:YLF Laser}

For the demonstration of a free running end pumped $\mathrm{Nd}$ :YLF laser we put the rod into a cavity with a length of $1.85 \mathrm{~m}$ as shown at the top of the figure 3 . The cavity use a flat output coupler with the reflectivity of $90 \%$ and a high reflector with a curvature of $5 \mathrm{~m}$. The lasing at 1053 $\mathrm{nm}$ is insured by the correct orientation of the Nd;YLF crystal using previous single-pass small signal gain measurement. And we also insert a brewster plate into the cavity which is used as our input and output port for our future MP structures. The existence of the brewster plate will also force the cavity lasing only at $1053 \mathrm{~nm}$ because it acts as a polarizer inside the cavity. The wavelength of the output laser radiation was also confirmed by a commercial optical spectrum analyzer. At the maximum one-sided pumping energy of $\sim 30 \mathrm{~mJ}$, the free-running output was $3 \mathrm{~mJ}$ in the multi-longitudinal, fundamental $\mathrm{TEM}_{00}$ mode, which is $10 \%$ of the pump energy delivered to the Nd:YLF rod surface. At the maximum two-sided pump energy of $\sim 60 \mathrm{~mJ}$, the free-running output was $9 \mathrm{~mJ}$ for the $\mathrm{TEM}_{00}$ mode, which is about $15 \%$ of the energy delivered to the rod surface. At the bottom of the figure 3 we plotted the resonator output vs the input energy for both side pump case. The inset is the image of $\mathrm{TEM}_{00}$ mode captured with a CCD camera. The highly symmetric image indicates a very good $\mathrm{TEM}_{00}$ mode. It's worth noting that we observed the good mode throughout the entire pump ranges without any transverse control inside the cavity. This could be explained by the fact that the end pumped cross section is smaller than the fundamental mode, which will function as an automatic mode control inside the cavity. [4] The optical to optical differential efficiency in our case is $15 \%$. In order to realize multi pass a Q-switch unit will be put in between the Brewster plate and the flat HR mirror. Initial test of the single pass operation with Q-switch unit resolve a gain of more than 4, which is already better than the performance of current MP structure. [1] 

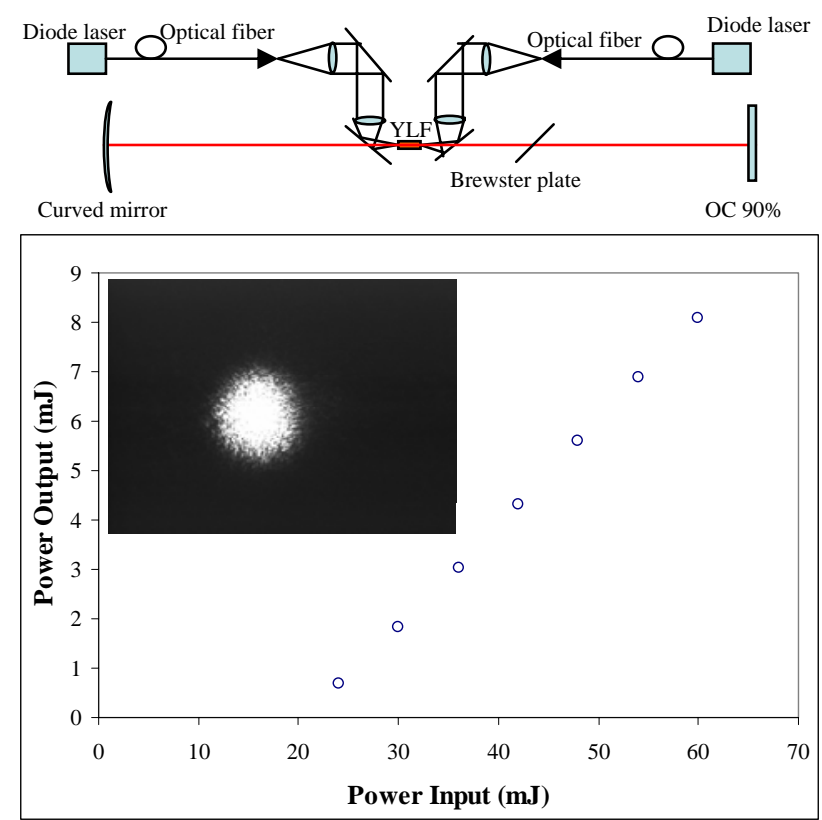

Figure 3: Top sketch is the end pumped $1053 \mathrm{~nm}$ Nd:YLF laser resonator layout. Bottom shows measured output energy vs. the pump energy. The inset shows the spatial profile from our $\mathrm{TEM}_{00}$ mode captured using a CCD camera.

\section{DISCUSSIONS}

For the future NML operation the power output is crucial for the generation of high quality e-beams. With the assumption of $1 \%$ quantum efficiency for the Cesium telluride $\left(\mathrm{Cs}_{2} \mathrm{Te}\right)$ photocathodes we will need $0.5 \mu \mathrm{J}$ for the generation of $1 \mathrm{nC}$ electrons. [6,7] If our optical efficiency is $15 \%$ as shown in current situation which will means that we will able to get $9 \mathrm{~mJ}$ for 1000 pulses, which corresponding to the single pulse energy of $9 \mu \mathrm{J}$. If we still follows the upgraded MP cavity with current flash lamp pumped 2-pass structure which will amplify the pulse more than 25 times we will get a pulse with the energy of more than $200 \mu \mathrm{J}$. [1] Considering the UV conversion efficiency is about $15 \%$ we will have a single $\mathrm{UV}$ pulse with the energy of $30 \mu \mathrm{J}$, which will produce more than $30 \mathrm{nC}$ electrons with the CsTe photocathodes even with the estimation of $50 \%$ loss during the transportation of UV. This means that $15 \%$ efficiency will be enough for the initial stage of the NML operation. To increase the efficiency of our resonator we can first increase the length of our lasing medium. At the same time it will benefit if we can switch our simple cavity design to a folded cavity design. A very high efficiency up to $54 \%$ was reported for the similar resonator using folded cavity design. [5]

\section{CONCLUSIONS}

In conclusion we have developed a diode laser end pumped Nd:YLF laser resonator. A good $\mathrm{TEM}_{00}$ mode with the energy of $9 \mathrm{~mJ}$ can be extracted with both sides pumped. The optical to optical efficiency is $15 \%$. This will be enough to support the initial operation of the newly developed ILC test facility in Fermi lab.

\section{ACKNOWLEDGEMENT}

The authors have benefited from many valuable discussions with Prof. A.C. Mellisinos from University of Rochester and Dr. Jianliang Li from Synopsys Inc. We also have to thank Dr. S. Schreiber for numerous suggestions.

This work is supported by Fermi Research Alliance LLC. under DE-AC02-07CH11359 with the U.S. DOE.

\section{REFERENCES}

[1] J. Li, R. Tikhoplav, A. C. Mellisinos "Performance of the upgraded laser system for the Fermilab-NIU photoinjector”, Nucl. Instru. and Meth. A. 564 (2006) 57

[2] J.E. Murray, "Pulsed gain and Thermal lensing of Nd:LiYF,", IEEE J. Quant. Electron. QE-19 (1983) 488.

[3] N.P. Barnes, M.E. Storm, P.L. Cross, M.W. Skolaut, "Efficiency of Nd Laser Materials with Laser Diode Pumping”, IEEE J. Quant. Electron. 26 (1990) 558

[4] F. Salin and J. Squier, "Gain guiding in solid-state lasers”, Opt. Lett. 17, 1352 (1992).

[5] "Efficient, end-pumped, 1053-nm Nd:YLF laser" LLE review, Volume 74, p. 120.

[6] I. Will, G. Koss, I. Templin "The upgraded photocathode laser of the TESLA Test Facility”, Nucl. Instru. and Meth. A ., 541 (2005) 467-477

[7] R. P. Fliller, H. Edwards, W. Hartung, "Time dependant quantum efficiency and dark current measurements in an RF Photocathode injector with a high quantum efficiency cathode”, Pac05, Knoxville, USA. 